\section{Die Talgabelung von Sargans}

\author{
In memoriam Dr. h. c. Heinrich Seitter
}

\section{Geologisch-tektonischer Überblick}

Die Umgebung von Sargans gehört geologisch und morphologisch zu den bedeutsamsten der ganzen Alpen. Durch das axiale Abtauchen der helvetischen unter die penninischen und ostalpinen Decken treten alle tektonischen Haupteinheiten der Alpen auf engstem Raum zusammen. Ebenso finden sich sub- und ultrahelvetische Elemente (Abb. 1).

Das Westende der ostalpinen Decken ist ein Erosionsrand, aber weder die ostalpinen noch die penninischen Decken bildeten einen kontinuierlichen Deckel über den helvetischen.

Talgabelungen sind ausgesprochen selten. Wo sie auftreten, lassen sie sich weder fluvial noch glazial erklären; sie sind tektonisch bedingt. Sie existierten bereits vor dem Eintreffen der Gletscher.

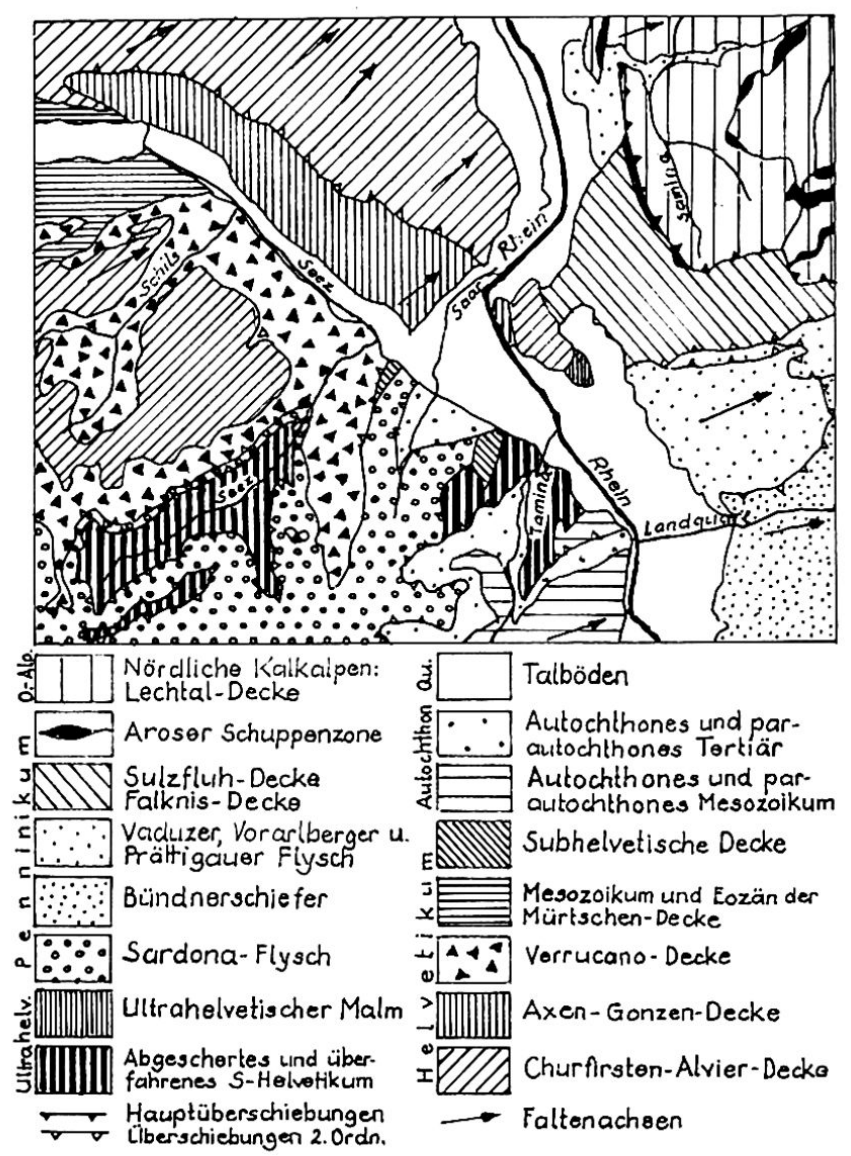

Abb. 1 Tektonische Übersicht der Talgabelung von Sargans.
Wohl wird das Abbrechen von Kristallin- und Sedimentpartien durch Fels- und Bergstürze auch um Sargans immer wieder eindrucksvoll vor Augen geführt (Abb. 2). Doch schon in den nördlichen Kalkalpen ist die Ausräumung der Täler durch Gletscher auf die Kaltzeiten beschränkt. Erratiker brachen als Sturzblöcke auf Gletscher und wurden von ihm verfrachtet. Dabei wurden karbonatische Blöcke aus älteren Kaltzeiten seither weitgehend gelöst.

Ein Vergleich des Deckenbaus von Wallis und Graubünden, wie ihn R. STAUB bereits 1917 postuliert und im «Bau der Alpen" (1924) seiner Zeit weit voraus dargetan hat, wurde nicht in erster Linie durch die erosive Tätigkeit im zentralen Bereich der Tessiner Kulmination und des Gotthard-Gebietes unterbrochen. Die Decken im Osten und Westen lagen viel näher beisammen. Sie glitten nicht nur nach Norden bzw. Nordwesten ab, sondern stets nach dem größten Gefälle. Dies gilt nicht nur großtektonisch für die penninischen und unterostalpinen Decken des Wallis und von Südbünden, sondern auch für die viel bescheideneren östlichen helvetischen Kalkalpen, wo sich das Ab- und Auseinandergleiten ihrer höheren Stockwerke, der Churfirsten-Alvier-Abfolge, von ihrer Verrucano-Unterlage modellhaft zeigt (A. HEIM \& J. OBERHOLZER 1917K, R. HELBLING 1938, J. OBERHOLZER 1942K, 1984 K). Die höheren Stockwerke glitten in den Churfirsten nach Norden, in der Alvier-Gonzen-Kette nach Nordosten ab. Zwischen der bei Murg stirnenden Verrucano-Abfolge und der nordwärts gefahrenen Mürtschenund Churfirsten-Stirn öffnete sich ein Tal, bildete sich ein erster Walensee. Das Seeztal entwickelte sich durch seitliches Abgleiten des bereits primären Faltenstapels des Jura-Stockwerkes von der Verrucano-(Trias-)Unterlage im Südwesten. Walensee-Talung und Seeztal waren so schon tektonisch angelegt; es hatten sich Zerrspalten gebildet. Diese wurden wohl durch Frost und kaltzeitliche Gletscher überprägt und etwas erweitert, aber nur unbedeutend übertieft. Die Wirkung des Eises wurde vor allem in den erosiv wirksamen Vorstoßphasen durch mündende Seitengletscher (Schils-, Murg- und vor allem Linthgletscher) erheblich gebremst. Das Weißtannental verdankt seine Entstehung dem frontalen Auseinanderbrechen der Flysch-Unterlage und der darüber gefahrenen starren Verrucano-Platte (Abb. 1).

René Hantke, Prof., Dr., Geologisches Institut der ETH und Universität Zürich, Sonneggstr. 5, CH-8092 Zürich 
Östlich des Rheins erhebt sich - an einem Grenzblatt, einer Blattverschiebung in der Gonzen-Decke, im Süden etwas zurückgeblieben, aber kaum mehr weiter axial abfallend - der Fläscherberg aus Gesteinen in süd- und ganz im Südosten in ultrahelvetischer Fazies.

Über der Verbindung von Prättigauer und Vaduzer Flysch erhebt sich die Falkniskette. Sie besteht aus einer in Falten gestauchten mittelpenninischen Jura-KreideAbfolge (D. TRUMPY 1916K). Weiter im Osten und weiter im Norden setzt sich über einer schmächtigen tiefmeerischen Ophiolith-Radiolarit-Abfolge, der Rätischen Dekke G. STEINMANNS, die ostalpine Lechtaldecke mit Schesaplana und Drei Schwestern ein.

Die Vorstellung, daß die Klippen - Grabser und Zentralschweizer Klippen, Préalpes Romandes - nur bescheidene Reste einer einst über die ganzen Westalpen sich erstreckenden, mehr oder weniger zusammenhängenden Decke erstreckt hätte und dann bis auf diese abgetragen worden wäre, bedarf ebenfalls einer Korrektur (HANTKE 1991).

\section{Morphologische Thesen im Lichte neuerer Forschungsergebnisse}

Mehrere morphologische Thesen - Übertiefung, Terrassen- und Trogtalbildung, rückschreitende Erosion - sind meist unbesehen von Generation zu Generation übernommen worden. Viele gehen auf die Frühzeit der Geologie, auf die Zeit vor der Deckentheorie, zurück. Und selbst diese ist noch immer im Wandel begriffen: Sie hat den Neuerkenntnissen aus Geologie, Geophysik, Paläontologie und Morphogenese stets Rechnung zu tragen. Leider wird sie noch zu oft als rein statisch betrachtet. In ihren inneren, kleinradigen Ablagerungsgebieten waren die Gesteinsserien flächendeckend. Bei ihrer Ausscherung und beim Vorgleiten über untertauchende Krustenelemente in äußere, großradigere Bereiche brachen sie auseinander. Dadurch bildeten sich bereits primär ohne jede erosive Einwirkung - Quertäler und beim Auseinandergleiten der einzelnen Stockwerke Längstäler. Dies betrifft neben der Klippendecke der Zentralschweiz

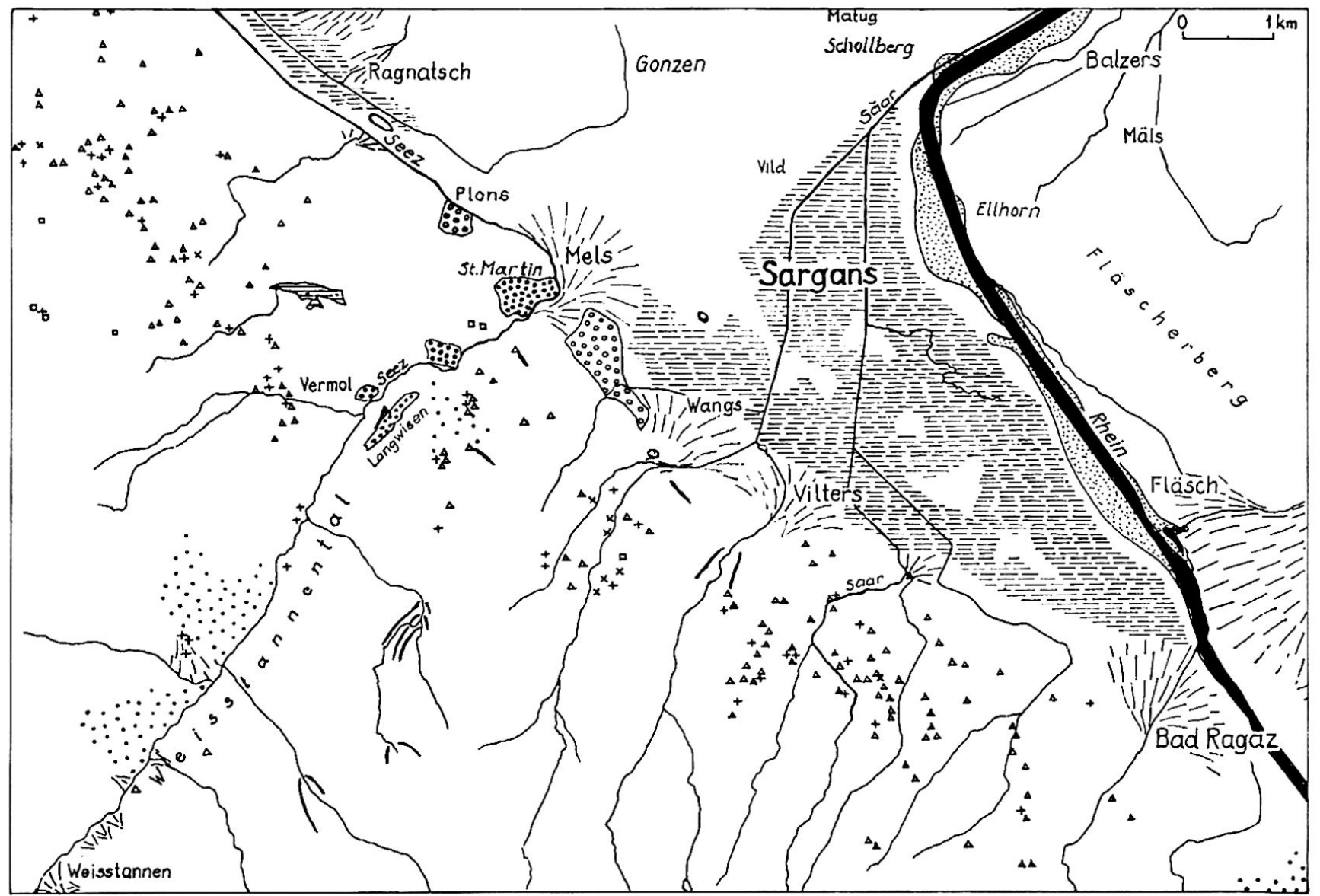

Erratiker:

- Melser Sandstein

$\times$ Verrucano

- Punteglicis-Granit

- Andere kristalline

Bündner Gesteine
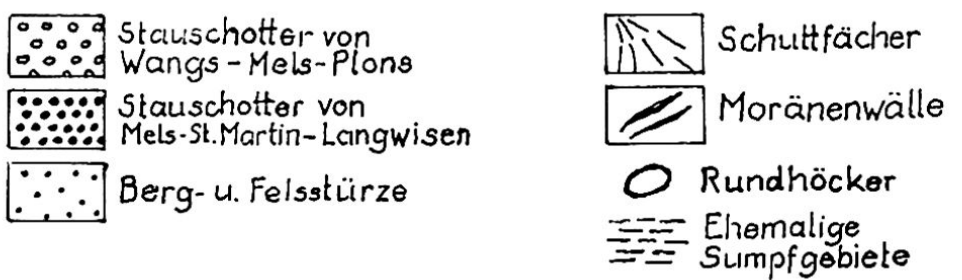

Abb. 2 Quartärgeologische Skizze der Talgabelung von Sargans. 
auch die helvetischen Decken, wo sich neben bedeutenden Querstörungen, Grenzblättern (Urner See, Bisistal, Linthtal), auch decken- und schuppentrennende Längstäler gebildet hatten.

\section{Die Anteile von Rhein-Gletscher und Tektonik bei der Talbildung}

Daß in Kühl- und Kaltzeiten der bei Sargans sich gabelnde Rheingletscher zur letzten Ausgestaltung der Talflanken und zur Schleifung der Talsohle beigetragen hat, zeigt sich mannigfach. Da Rundhöcker nicht nur aus resistenten Gesteinen bestehen, verdanken sie ihre Anlage einer Schertektonik. Zudem sind im Alpenrheintal selbst ihre jüngsten Kreideschichten im Luv des fließenden Eises noch erhalten, so daß dessen Wirkung sorgsam zu überprüfen ist. Um Sevelen sind es Wang-Schichten, rheintalabwärts, wo diese aus faziellen Gründen ausfallen, Amdener Schichten und Seewer Kalk, welche die jüngsten Schichten bilden.
Im oberen Teil taucht die verfaltete Kreideabfolge der Alvierkette unter den Prättigauer, Vaduzer und Voralberger Flysch. Dazwischen bildete sich ein Stück Alpenrheintal. Über der mittelpenninischen Falknisdecke folgt die Aroser Zone und dann die in Schollen zerlegte ostalpine Lechtaldecke, welche die Gipfel der Schesaplana und der Drei Schwestern aufbaut. Neben dem Abgleiten der höheren Stockwerke waren für die Talbildungen Verscherungen verantwortlich. Solche manifestieren sich im Seeztal zwischen den Liasrippen von Gräpplang und St. Georgen. Im Seeztal zeichnet sich quer zu den Faltenstrukturen ein Grenzblatt ab; die beiden Talseiten würden sich auch mit ihrer ursprünglichen Gesteinsfüllung nicht entsprechen. In der Ebene tritt der Hügel des Tiergarten und bei Sargans jener von Castels aus den Alluvionen empor. Auffallend ist sodann der steile Abfall des Sarganser Schloßhügels und seiner Fortsetzung seezabwärts, der Tschuggenkette. Diese Fakten - zusammen mit den Bohrresultaten - deuten darauf hin, daß talparallele Klüfte und Scherung am Werk waren und dadurch ein Relief mit Rinnen und Tälchen entstand (Abb. 3).

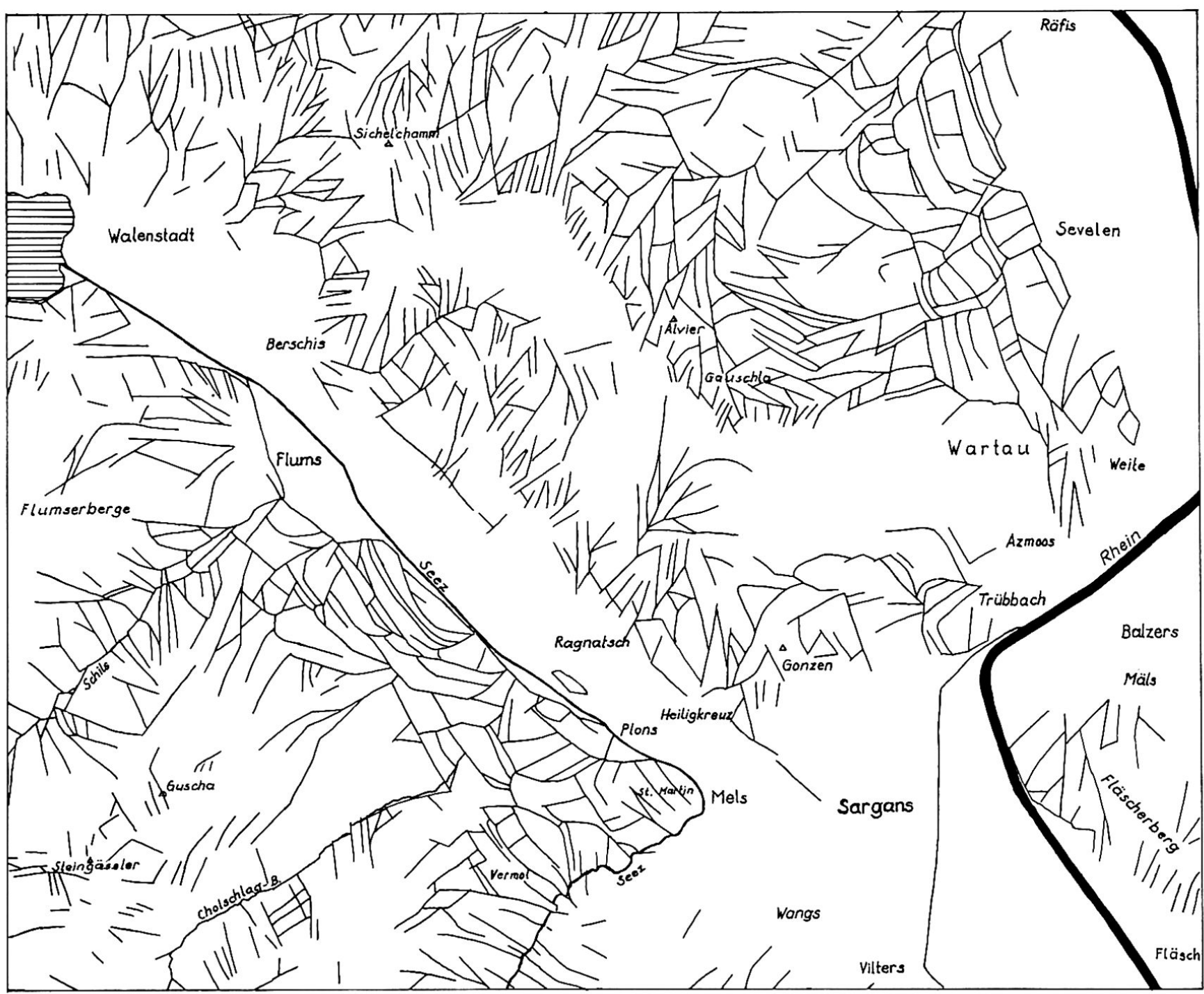

Abb. 3 Scherstörungen im Bereich der Talgabelung von Sargans. 
Zugleich ergaben die Bohrungen für Grundwasser, daß an der Basis nicht Grundmoräne, sondern Schotter ansteht. Die Steilabfälle der tiefsten Wände der Alvierkette mit dem Sarganser Schloßberg setzen sich unter den Schuttfächern und Talalluvionen fort (M. KOBEL. schr. Mittl., 1992, G. P. JUNG, schr. Mittl., B. MÜLLER 1992). Im Gebiet des Stadtgrabens von Sargans zeichnet sich zudem noch ein weiterer, nicht mehr über die Talalluvionen emporragender Rundhöcker ab (Abb. 4). Auch auf der Südwestseite des Seeztales versteilt sich das Gehänge gegen das Tal; zugleich stellen sich zahlreiche oft talparallele Störungen ein. Diese Fakten wurden stets als glazial übertieft gedeutet, da Talbildungen nach der bisherigen Lehrmeinung nur durch Eintiefung durch fließendes Wasser oder Eis erfolgen konnten.

Rheintalabwärts, zwischen den Säntisketten, die gegen Osten in Staffelbrüchen zum Rhein abfallen, im Rheintal eine Senke nachzeichnen und gegen Osten teils steil etwa in der Hohenemser Falte - in den Vorarlberger Kreideketten aufsteigen, sind stets die tektonisch höchsten Elemente und zudem ihre jüngsten Schichtglieder noch erhalten.

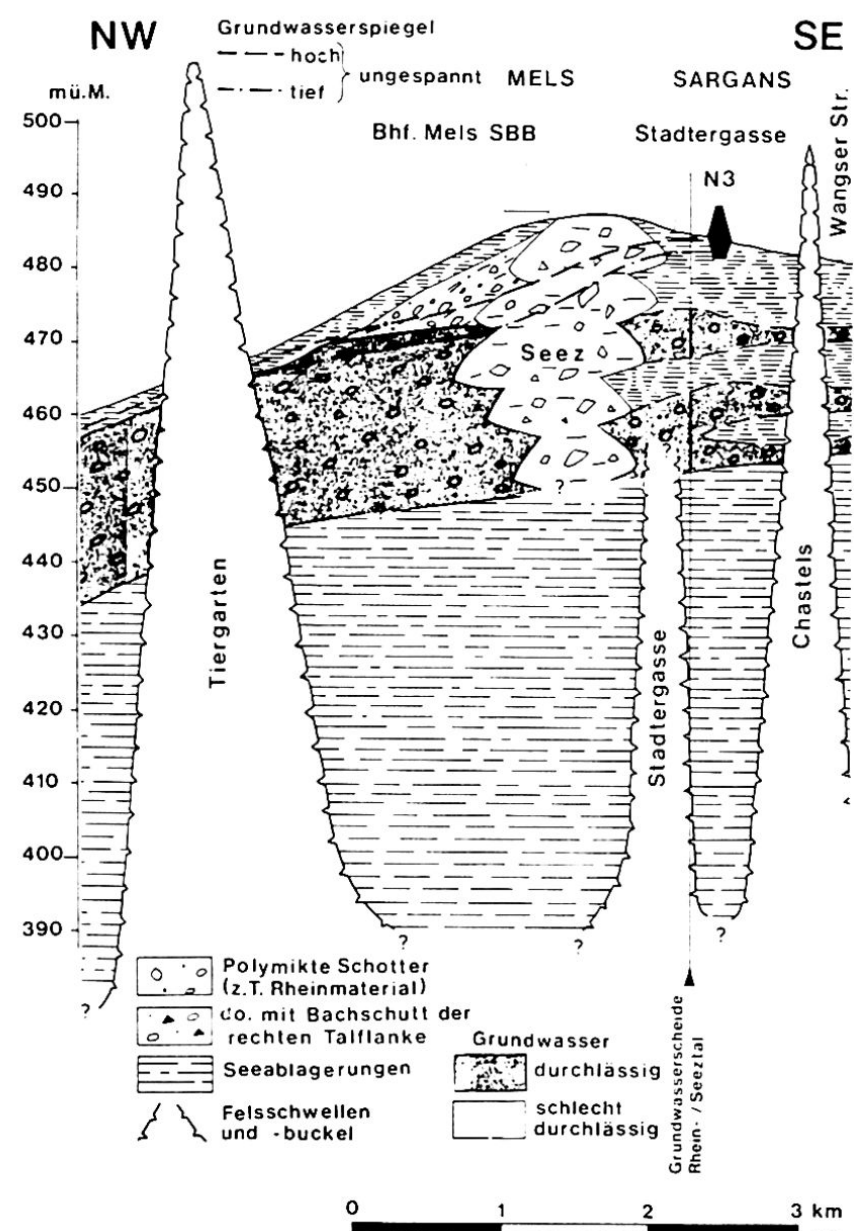

Abb. 4 Scherstörungen und Grenzblätter im Bereich der Talgabelung von Sargans, nach M. KOBEL, schr. Mitt.
Bei den Inselbergen zeigt sich, daß diese randlich durch Scherstörungen begrenzt werden. Bereits bei der Platznahme der Decken wurden diese in Teile auseinandergeschert. Das Alpenrheintal ist daher tektonisch angelegt, nicht als Grabenbruch in ein fertiges Deckengebäude; es existierte schon vor dem Eingleiten, der Platznahme der Decken und geht damit letztlich bereits auf die früheste Molassezeit zurück. Damals wurden in oligo- und miozänen Kühlzeiten bei tiefer Waldgrenze mächtige Schuttfächer geschüttet. Diese erfolgten in die orographisch tiefsten Bereiche. Bei der Platznahme der ankommenden helvetischen Decken wurde die zuvor dort lagernde, in eine ältere Talung geschüttete Molasse längs Gleitflächen ausgeschert und dachziegelartig übereinandergestaucht. Ihre frontalste Partie wurde dabei über die gegen Süden sich bewegende, flachliegende, schon in einer Frühphase sich aufrichtende und sich verscherende Vorlandmolasse geschoben (Toggenburg-Schwägalp-Weißbad).

\section{Der Ur-Rhein nach dem Anrücken der helvetischen Decken}

Während sich der Urrhein als Folge von Muren bis ins jüngste Miozän gegen Nordwesten wandte und dort, vor$\mathrm{ab}$ in Kühlphasen bei tieferer Waldgrenze, einen großen, aber recht flachen Schuttfächer, den Hörnlifächer, aufbaute, wurde ihm mit dem Anrücken der helvetischen Decken dieser Weg versperrt; er mußte sich einen neuen Austritt durch den veränderten Alpenrand suchen. Einen solchen fand er durch das mittlere Alpenrheintal. Aufgrund von Tiefbohrungen (Dornbirn $336 \mathrm{~m}$, Hohenems $592 \mathrm{~m}$ ) ist dieses recht tief. Früher wurde es als "glazial ausgeräumt», als «übertieft», betrachtet. Doch wiederum ist es nicht Grundmoräne, welche die tiefste Füllung bildet. Würmzeitliche Grundmoräne mit gekritzten Geschieben wurde bei Hohenems in einer nur wenige $100 \mathrm{~m}$ entfernt niedergebrachten Bohrung bereits in $16 \mathrm{~m}$ Tiefe angetroffen. Auch das Alpenrheintal ist tektonisch vorgezeichnet. Die Arbeit des Rheingletschers bestand darin, von den Seiten niedergebrochenen Gesteinsschutt und vom Untergrund weggesprengtes Schürfgut weiterzutransportieren. Daß er dabei den Felsgrund schliff, wird an Geländekanten und Rundhöckern offenkundig. Selbst die Tiefbohrung von Hohenems hat mit $592 \mathrm{~m}$ kaum den tiefsten Punkt getroffen; doch zeigten seismische Untersuchungen, daß sie recht tief reicht.

Nach den jüngsten Ablagerungen, die am Hörnli nach den Floren- und Faunenuntersuchungen von TH. BOLLIGER \& M. EBERHARD (1989) bis ins jüngere Mittelmiozän reichen, schienen in der Nord-Schweiz jüngstmiozäne bis ältestpleistozäne Sedimente zu fehlen. Sie wurden meist als nicht abgelagert oder als abgetragen betrachtet. $\mathrm{Da} ß$ in den steilen Gipfelpartien des Hörnligebietes Bereiche abgetragen worden sind, ist offenkundig. Ob dies aber dort sämtlicheSedimente seit dem jüngeren Miozän betrifft, ist recht unwahrscheinlich. Mit der Anlage der neuen Entwässerung an der Mio-/Pliozän-Wende durch 
das neu entstandene Alpenrheintal sind auch die Sedimente nicht mehr auf den Höhen des Hörnliberglandes abgelagert worden - dort wurde effektiv seither abgetragen -, sondern in den tiefsten Bereichen des jungen Alpenrheintales. Die basalen Sedimente der Hohenemser Bohrung über dem Vorarlberger Flysch erweckten denn auch einen kühlzeitlichen Eindruck.

\section{Die jüngeren Kaltzeiten um Sargans und Tertiärrelikte}

In den größten Kaltzeiten reichte das Eis des an der Diffluenz von Sargans sich gabelnden Rheingletschers bis auf die Höhe des Gonzen. Noch in der Würmeiszeit stand dieser Eckpfeiler aus helvetischen Jurakalken bis auf eine Höhe von fast 1700 m unter Eis.

F. SAXER (1969) postulierte, daß das aus dem Weißtannental austretende Seezeis dem Rheineis den Weg durch die Seez-/Walenseetalung weitgehend verbarrikadiert hätte. Nur etwa 2 bis $3 \%$ des Rheineises hätten den Weg durch das Seeztal gefunden. Eine Überprüfung ergab allerdings, daß gut 70\% durch das Alpenrheintal, aber immerhin noch knapp 30\% durch die Walenseetalung abgeflossen waren (HANTKE 1970).

Aufgrund von Erratikern aus dem Bündner Oberland drang das Rheineis in den Hochglazialen von Mels kräftig ins Weißtannental ein und drängte das Seezeis bis fast nach Weißtannen zurück. Bereits J. OBERHOLZER (1920 K, 1933) fand nördlich der Mühli, 2,5 km vor Weißtannen, drei Puntegliasgranite und 1,5 bzw. knapp $1 \mathrm{~km}$ vor dem Dorf zwei weitere Bündner Oberländer Blöcke. Sie bekunden, daß das Seezeis noch in den Maximalständen der Würmeiszeit vom Rheingletscher kräftig zurückgedrängt wurde. Das Seezeis konnte damals nurganz an der linken Talflanke austreten. Für einen Transport über den Kunkelspaß ins untere Calfeisental und über den Heitelpaß $(2388 \mathrm{~m})$ ist dieser selbst in den größten Eisständen um über $200 \mathrm{~m}$ zu hoch.

Von den Rückschmelz- und Wiedervorstoßlagen des Spätwürms zeichnet sich nach den Ständen von Schänis/ Weesen, der sich dort in einer randlichen Schotterflur, randlichen Moränenresten, der Stirnmoräne von Biäsch und im untersten Walensee durch das bei der Linthkorrektion abgetragene und angelandete Inselchen von Hüttenböschen abzeichnet, das sich noch auf der Schweizer Karte von J. J. SCHEUCHZER (1712K) findet. An der Mündung des Weißtannentales haben sich auf der rechten Seite eine Stauterrasse und Wallreste erhalten. Um Sargans zeichnen sich diese älteren Stände von Schänis/Weesen im vorderen Weißtannental in der Abflußrinne Plattmol-Chapfensee und in der Stauterrasse von Langwisen ab. Diese wird von kurzen Moränenwällen begleitet, von denen jene des Seezgletschers um 890-880 m noch erhalten, während jene des Rheingletschers weitgehend abgetragen worden sind. Eine tiefere, ebenfalls tektonisch vorgezeichnete Rinne verläuft südwestlich des Furtschchopf. Über dem linken Ausgang des Weißtannentales, beim Hinteren und Vorderen Schlößli, ist sodann ein jüngerer Stand durch tiefere Schotterterrassen- reste belegt. Dieser Eisstand zeichnet sich auch bei Rüfi-St. Martin westlich von Mels und bei Plons im Seeztal ab. Er verrät dort mit einer Abflußrinne ein Zungenende unterhalb von Plons, am Tiergarten und bei Ragnatsch. Noch jüngere Stände geben sich durch Stauschotter westlich von Wangs, südöstlich von Mels und in Sargans zu erkennen (OBERHOLZER 1920K, 1933).

Die Churfirsten-Alvier-Kette, in etwas geringerem Maße auch die St. Galler Oberländer Alpen, zeichnen sich durch zahlreiche Tertiärrelikte aus, Pflanzen, die sich in diesen Gebieten über dem Taleis und unter feuchtigkeitsspendenden Firnkappen einfanden. In den Interglazialzeiten vermochten sie sich über dem jeweils eingewanderten Wald zu behaupten. Neben wenigen Endemiten sind dies meist krautige Alpenpflanzen, die heute von $2000 \mathrm{~m}$ bis zur Vegetationsgrenze vorkommen. Sie kommen mit einer kurzen Vegetationszeit aus und zeichnen sich durch Trockenresistenz aus (H. SEITTER \& R. HANTKE 1988).

\section{Der Sarganser Raum vom ausgehenden Spätwürm bis in die Römerzeit}

Die Zuschüttung des Seeztales erfolgte durch Muren, wohl Ausbrüche spätglazialer Moränenseen vor den Zungen der Seitengletscher. Dazwischen liegen Seesedimente: zunächst in der Fortsetzung vom Walensee bis zur Mündung des Schilstales und in einem weiteren Becken zwischen Schilsfächer, der einen See staute, und Seezmündung aus dem Weißtannental. In die Seen ergossen sich die Schuttfächer von Widen- und Berschner Bach, Röll-, Cholschlager- und Ragnatscher Bach.

Als altsteinzeitliche Jagdhöhle diente das Drachenloch ob Vättis. In der Jungsteinzeit, um 3000 v. Chr., bauten sich Bauern auf den Höhen von Castels oberhalb Mels und auf Severgal ob Vilters einfache Pfostenhütten. Die Plätze waren bis in die Bronzezeit besiedelt, lieferten doch beide Orte auch Keramikscherben und Bronzegegenstände. Aus der späteren Urnenfelderzeit stammen Funde von Heiligkreuz (B. FREI 1966). In der frühen Eisenzeit drangen die Räter bis ins Sarganserland vor. Noch in römischer Zeit reichte der Walensee seezaufwärts bis an den Fuß des St. Georgsberg bei Berschis. Neben den nachrömischen Schiffsstationen Terzen, Quarten und Quinten, der dritten, vierten und fünften am Walensee (die beiden ersten dürften in Berschis und Walenstadt gelegen haben), sind es vor allem Wachttürme, welche die Verkehrswege von Chur nach Zürich und weiter sicherten: Schänis-Biberlichopf, Betlis-Stralegg, Filzbach-Voremwald und Walenstadt. Bei Weesen stand auf dem Inselchen Hüttenböschen im untersten Walensee ein gallo-römischer Tempel. Auf dem Georgsberg liegt ein spätrömisches Refugium; in Flums fanden sich Gebäudereste, und von Sargans-Malerva wurden Reste eines Gutshofes ausgegraben (W. DRACK \& R. FELLMANN 1991). Die Sarganser Ebene, die Sarganser, Melser und Vilterser $\mathrm{Au}$ lagen mindestens bei Hochwasser des Rheins noch unter Wasser (Abb. 2). 


\section{Der Sarganser Raum im Mittelalter und Neuzeit}

Vom Bodensee her drang die Germanisierung nur zögernd südwärts vor. Lange Zeit lag die Grenze zum Romanischen am Montlinger Berg, einem alten Kulturzentrum über der Rheinebene. Dies belegen Orts- und Flurnamen. Auch das Seeztal und der Walensee waren «welsch». Walenstadt (= Stätte der Welschen, der Rätoromanen) wird erstmals 831 erwähnt, so daß der See damals wohl noch bis dorthin reichte - heute endet er mehr als $1 \mathrm{~km}$ weiter westlich. Mels und Sargans - Senegaune traten urkundlich schon 765 auf. Nur wenig früher, 762, ist Pfäfers, eines der bedeutendsten Klöster Rätiens, beurkundet.

Im Verkehrsnetz war die Talgabelung von Sargans schon seit römischer Zeit von Bedeutung. Das Schloß wurde erst 1282 als Sitz der Grafen von Werdenberg-Sargans erwähnt. Von 1483 bis 1798 war es Amtssitz der Landvögte der 7-, ab 1717 8örtigen Eidgenossenschaft.

Das ehemalige Zungenbecken war teilweise noch bis tief ins $20 \mathrm{Jh}$. von Ried eingenommen. Über dem feinen Schlick des bei Hochwasser überflutenden Rheins hielt sich die Nässe außerordentlich lange; die Auflage humussäurereicher Pflanzensubstanz schritt nur langsam voran. Dies ermöglichte einer vielfältigen kostbaren Riedflora und ihrer Begleitfauna, lange Zeit zu überdauern. Grund genug, ihnen wenigstens in kaum meliorierbaren Gebieten ein Überleben zu sichern.

Da der Rhein vom Ellhorn direkt zum Fuß des Schollberg floß und dort noch die Saar mündete, war die Stelle lange Zeit für den Verkehr unpassierbar. Der alte linksufrige Rheintalweg führte daher von Sargans über Vild-Matug-Azmoos, rechtsrheinisch von Maienfeld über die Luziensteig nach Balzers. Erst mit der Verbauung des Rheins im Bereich der Saar-Mündung konnte die Straße am Schollbergfuß entlang nach Trübbach geführt werden.

Meliorationen - «Verbesserungen» - wurden propagiert, um die Ernährungsgrundlage der stark angestiegenen Bevölkerung bei sinkendem Streuebedarf sicherzustellen. Doch wird dadurch das biologische Gleichgewicht gestört. 1968 wurden zu leichtfertig die letzten Riedlandschaften geopfert.

\section{Literatur}

BOLLIGER, TH., EBERHARD, M. (1989): Neue Faunen- und Florenfunde aus der Oberen Süßwassermolasse des Hörnligebietes (Ostschweiz). Vjschr. natf. Ges. Zürich 134/1.

DRACK, W., FELLMANN, R. (1991): Die Schweiz zur Römerzeit. Zürich und München (Artemis).

FREI, B. (1966): Zeugen der älteren Urnenfelderzeit aus dem Bereich des oberen Alpenrheins. Helv. Antiqua (Festschrift Emil Vogt) 1966.

HANTKE, R. (1961): Tektonik der Helvetischen Kalkalpen zwischen Obwalden und dem St. Galler Rheintal. Vjschr. natf. Ges. Zürich 106/2.
HANTKE, R. (1970): Die Diffluenz des würmeiszeitlichen Rheingletschers bei Sargans und die spätglazialen Gletscherstände in der Walenseetalung und im Rheintal. Vjschr. natf. Ges. Zürich 115/1.

HANTKE, R. (1980): Eiszeitalter, Band 2. Thun (Ott).

HANTKE, R. (1987): Zur Tal- und Reliefgeschichte des Churfirsten-Alvier-Gebietes (Kanton St. Gallen). Geogr. Helv. 1987/ 4: $159-168$.

HANTKE, R. (1991): Landschaftsgeschichte der Schweiz und ihrer Nachbargebiete. Thun (Ott).

HEIERLI, H. (1984): Die Ostschweizer Alpen und inr Vorland; Säntismassiv, Churfirsten, Mattstock, Alviergruppe, Appenzeller Molasse. Samml. geol. Führer 75. Berlin, Stuttgart (Borntraeger).

HELBLING, R. (1938): Zur Tektonik des St. Galler Oberlandes und der Glarner Alpen. Beitr. geol. Karte Schweiz N. F. 76.

KOBEL, M. (1992): Rhein- und Seeztal. In: Blatt Toggenburg, Hydro-geol. Atlas Schweiz.

MÜLLER, B. (1992): Zur Quartärgeschichte des Seeztales (Kt. St. Gallen). - In Vorb.

OBERHOLZER, J. (1933): Geologie der Glarner Alpen. Beitr. geol. Karte Schweiz, N. F. 28.

SAXER, F. (1969): Die Differenz des Rheingletschers bei Sargans. ECl. geol. Helv. 57/2: 604-607.

SEITTER, H., HANTKE, R. (1988): Mögliche jüngsttertiäre Florenrelikte in der Speer-Churfirsten-Alvier-Kette und im St. Galler Oberland. Ber. st. gall. natw. Ges. 83: 129-160.

STAUB, R. (1917): Das Äquivalent der Dentblanchedecke in Bünden. Vjschr. natf. Ges. Zürich 62/1-2.

STAUB, R. (1924): Der Bau der Alpen - Versuch einer Synthese. Beitr. geol. Karte Schweiz, N. F. 192.

\section{Geologische und alte topographische Karten}

HEIM, A., OBERHOLZER, J. (1917K): Geologische Karte der Alvier-Gruppe 1 : 25 000. Geol. Spez.-Karte 80. Schweiz geol. Komm. Basel.

JUNG, G. P. (1981): Sarganserland 1845 und 1978, 1:50 000. In: Schweizer Weltatlas mit noch unveröffentlichtem Kommentar. Zürich (Lehrmittelverlag).

OBERHOLZER, J. (1920K): Geologische Karte der Alpen zwischen Linthgebiet und Rhein - Flumser Alpen, Graue Hörner, Ringelspitz, Calanda. 1:50 000. Geol. Spez.-Karte 63. Schweiz. geol. Komm. Basel.

OBERHOLZER, J., et al. (1942K): Geologische Karte des Kantons Glarus - Geol. Spez.-K. 117. Schweiz. geol. Komm. Basel (Neudr. 1984 K).

SCHEUCHZER, J. J. (1712K): Nova Helveticae Tabula geographica..., etwa $1: 238000$, Zürich.

SPICHER, A. (1980K): Tektonische Karte der Schweiz. Schweiz. geol. Komm. Basel.

TRÜMPY, D. (1916K): Geologische Karte des Falknis. $1: 25$ 000. Geol. Spez.-Karte 79. Schweiz. geol. Komm. Basel.

\section{Dank}

Den Herren Dr. G. Jung, Bad Ragaz, Dr. M. Kobel, Freienbach, und $\mathrm{H}$. Wäspi, Winterthur, sei für die Mithilfe bestens gedankt. 